\title{
An Integrated Picture Archiving and Communications System-Radiology Information System in a Radiological Department
}

\author{
M. Wiltgen, G. Gell, E. Graif, S. Stubler, A. Kainz, and R. Pitzler
}

In this report we present an integrated picture archiving and communication system (PACS)-radiology information system (RIS) which runs as part of the daily routine in the Department of Radiology at the University of Graz. Although the PACS and the RIS have been developed independently, the two systems are interfaced to ensure a unified and consistent long-term archive. The configuration connects four computer tomography scanners (one of them situated at a distance of $1 \mathrm{~km}$ ), a magnetic resonance imaging scanner, a digital subtraction angiography unit, an evaluation console, a diagnostic console, an image display console, an archive with two optical disk drives, and several RIS terminals. The configuration allows the routine archiving of all examinations on optical disks independent of reporting. The management of the optical disks is performed by the RIS. Images can be selected for retrieval via the RIS by using patient identification or medical criteria. A special software process (PACS-MONITOR) enables the user to survey and manage image communication, archiving, and retrieval as well as to get information about the status of the system at any time and handle the different procedures in the PACS. The system is active $\mathbf{2 4}$ hours a day. To make the PACS operation as independent as possible from the permanent presence of a system manager (electronic data processing expert), a rule-based expert system (OPERAS; OPERating ASsistant) is in use to localize and eliminate malfunctions that occur during routine work. The PACS-RIS reduces labor and speeds access to images within radiology and clinical departments.

Copyright 01993 by W.B. Saunders Company

KEYWORDS: picture archiving and communication systems (PACS), radiological information systems (RIS).

$\mathbf{T}$ HE DEVELOPMENT of digital imaging methods such as computer tomography, magnetic resonance imaging, and digital subtraction angiography has led to the possibility of digital image transfer via network from image

From the Institut für Medizinische Informatik, Statistik und Dokumentation, Graz, Austria.

Supported by the Österreichische Nationalbank.

Address reprint requests to $M$. Wiltgen, Institut für Medizinische Informatik, Statistik und Dokumentation, Auenbnggerplatz 9/III, 8036 Graz, Austria.

Copyright $₫ 1993$ by W.B. Saunders Company

0897-1889/93/0601-0001\$03.00/0 acquisition modalities to viewing stations and to store images in a digital way. Such systems are called picture archiving and communication systems (PACS). The advantages of PACS are reduced costs for film and archive space, faster access to the images and image processing capabilities of the viewing consoles. The necessity, the advantages, the costs, and the design of such systems have been discussed in various publications. ${ }^{1-12}$ We present the development of and our experiences with a PACS which is in daily routine use in a radiological department. The experiences come from the use of the PACS over a period of four years. Our main developments are tools for the management and survey of the PACS, the detection and recovery of errors that occur during routine operation, and the development of software for image display consoles based on personal computers (PCs). From our point of view, the PACS is a part of a radiological information system (RIS) and it is arbitrary which function belongs to the RIS and which to the PACS. The PACS is linked with an RIS named AUtomatic Report Analysis (AURA) which manages the archive. AURA was developed in the Department of Radiology at the University of Graz. ${ }^{13-15}$

\section{THE PACS IN THE DEPARTMENT OF RADIOLOGY}

In collaboration with Siemens (Erlangen, Germany), the PACS was installed step-by-step in the Department of Radiology of the University of Graz. This PACS is used daily for the archiving, retrieval of images and image exchange within the Department of Radiology. ${ }^{16-18}$

\section{System Configuration}

In the Department of Radiology, three CT scanners, a DSA unit, an evaluation console, an archive with two drives for optical disks, a PACS diagnostic console with three high-resolution $(1,000 \times 1,000$ pixels) monitors (Micro VAX II; Digital Equipment Corp, Maynard, MA) and an image display console (DRC 101; Siemens, 
Germany; console computer is a SUN-workstation) are connected by a local area network (CT-NET) which is linked with a second network (LKH-NET) (Fig 1). Both networks are based on Ethernet (10 Mbit/second) and have a bus topology. The LKH-NET, which is a hospital-wide network, connects a CT scanner, a Mevaplan, an Evaluskop (Siemens) in radiotherapy which is located at a distance of approximately $1 \mathrm{~km}$ from the radiological department, an MRI scanner, an image viewing console (based on PC) and several terminals of the RIS.
The computer of the diagnostic console, a Micro VAX II, is the gateway between the two networks. For the connection, coaxial cables (thick and thin wire Ethernet) are used inside the departments and fiber optic cables between the buildings. The CT images are initially in a proprietary format, and for the communication, the CT-NET (a subset of DECnet, Digital Equipment Corp, Boston, MA) protocol is used. For the transmission of the MRI images the DECnet protocol is used. The images are simply copied via the DEC utility program.
Fig 1. PACS configuration at the Department of Radiology of the University of Graz. The PACS includes four CT-scanners, an MRI-scanner, a DSA, two evaluation consoles (EVA), a radiotherapy planning system (Mevaplan). a diagnostic console (PDM), an image display console (DRC 101). an image viewing console (based on $\mathrm{PC}$ ) and an archive with two drives for optical disks. The PACS is interfaced with a radiological Information system (RIS).

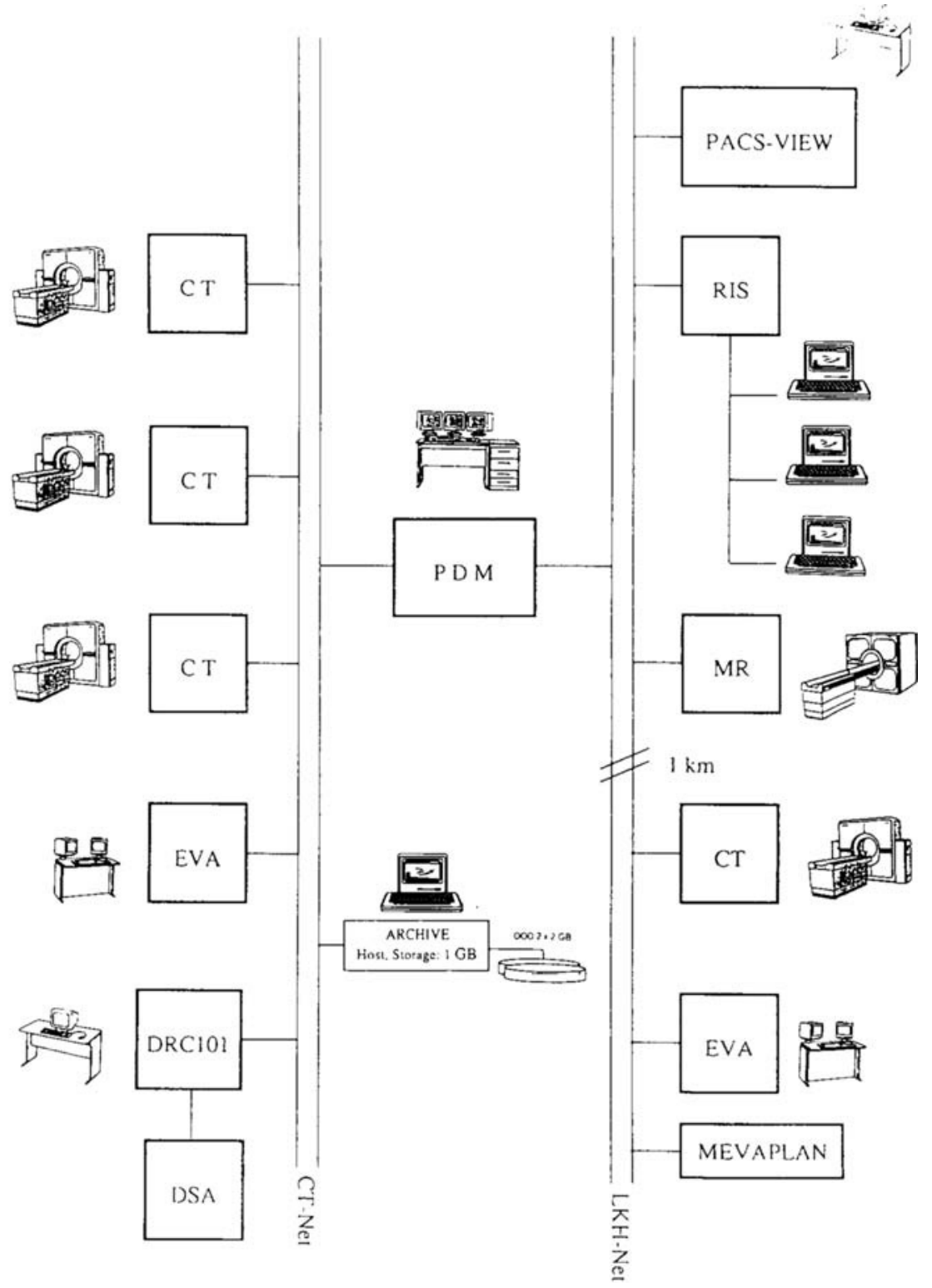




\section{Storage Structures}

\section{Long-time Archive}

Optical disks (2 Gbytes) are used for the long-time archiving of examinations. The management of the optical disks is performed by the RIS. The images are archived with a resolution of $256 \times 256$ pixels and compressed by a factor of 2. For the compression the Differential Pulse Code Modulation-Algorithm is used.

\section{Intermediate Magnetic Store}

Before the images are copied on optical disk and after the retrieving, the images are stored on magnetic disks (Fig 2). The images on this

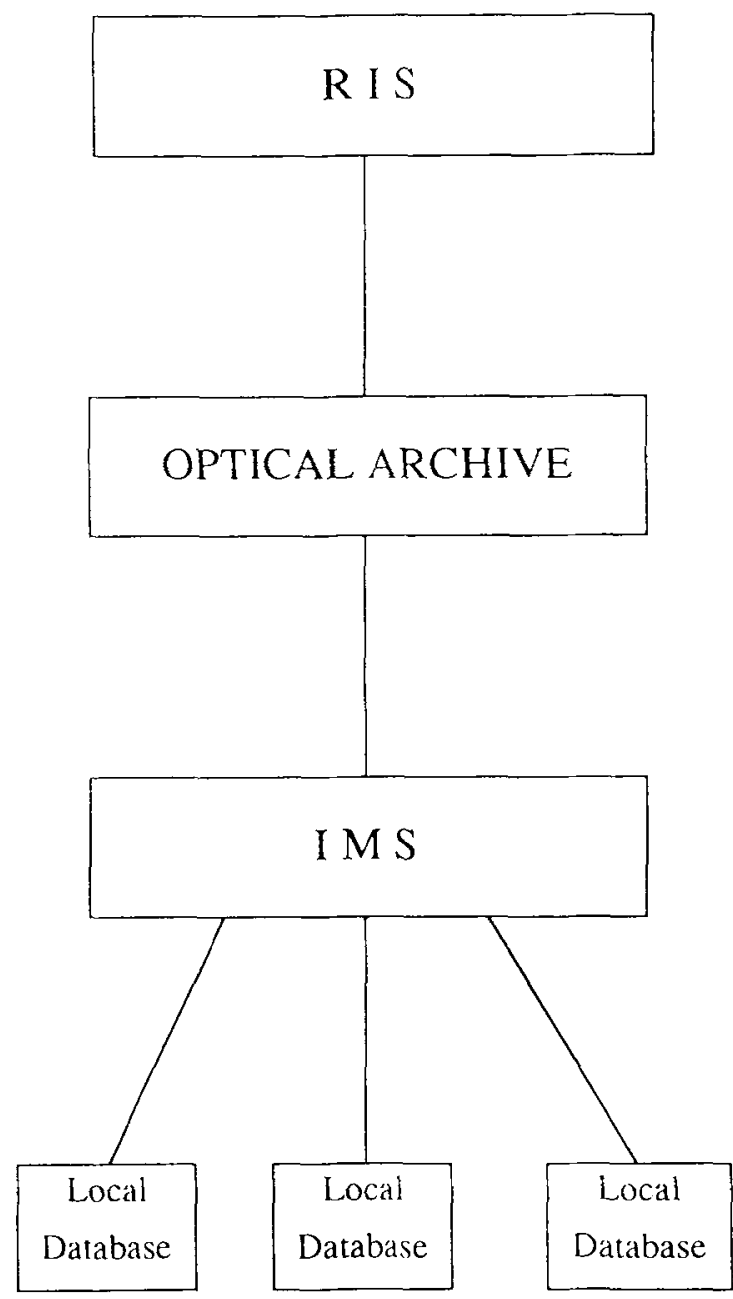

Fig 2. Storage structure: Optical disks are used as longtime archive. The disks are managed by the RIS. A magnetic store is used as intermediate store, the images on the magnetic store are managed by the IMS. At the viewing stations exist local databases for the access to the images. intermediate magnetic store are managed by the IMS data base (Image Management System). The IMS data base contains patient, examination, and image data and enables access to the images on the magnetic storage by patient data. The data are held in folder structures.

\section{Local Data Base}

There are local data bases at the viewing consoles that give the user at the various viewing consoles (diagnostic console, image display console) faster access to the images stored locally. These data bases are copies of the IMS.

\section{Software Components of the PACS}

\section{Software Process Structures}

The PACS is organized as a group of software processes that run in the background (Fig 3). The communication between the processes is administrated by the Siemens PACS-Net/SPI Toolkit, which complies with the American College of Radiology (ACR)-National Electrical Manufacturer's Association/International Society for Optical Engineers ${ }^{19}$ commands to request and transfer images. The processes remain in a waiting state until they are activated by commands from other processes. The process structure is distributed over several computers. Most operations in the PACS are done automatically by the software processes, which provide access to the images and image data.

There are processes for the conversion of images into the ACR-NEMA format, the transfer of images to local databases, the management of the local databases or the IMS database etc. The processes execute these tasks in the background while the user continues with other work. The sequence of processes operates as a pipeline: during the submission of the first image into IMS, the second image is already being converted into ACR-NEMA, and the third image is transferred over network. Thus the time needed to execute a task (archiving, retrieving) is much shorter then the sum of the times for each process.

\section{PACS-MONITOR}

A special software process (PACS-MONITOR) is used to handle and survey image communication, archiving, and retrieving in the PACS (Fig 4). The user can get information 


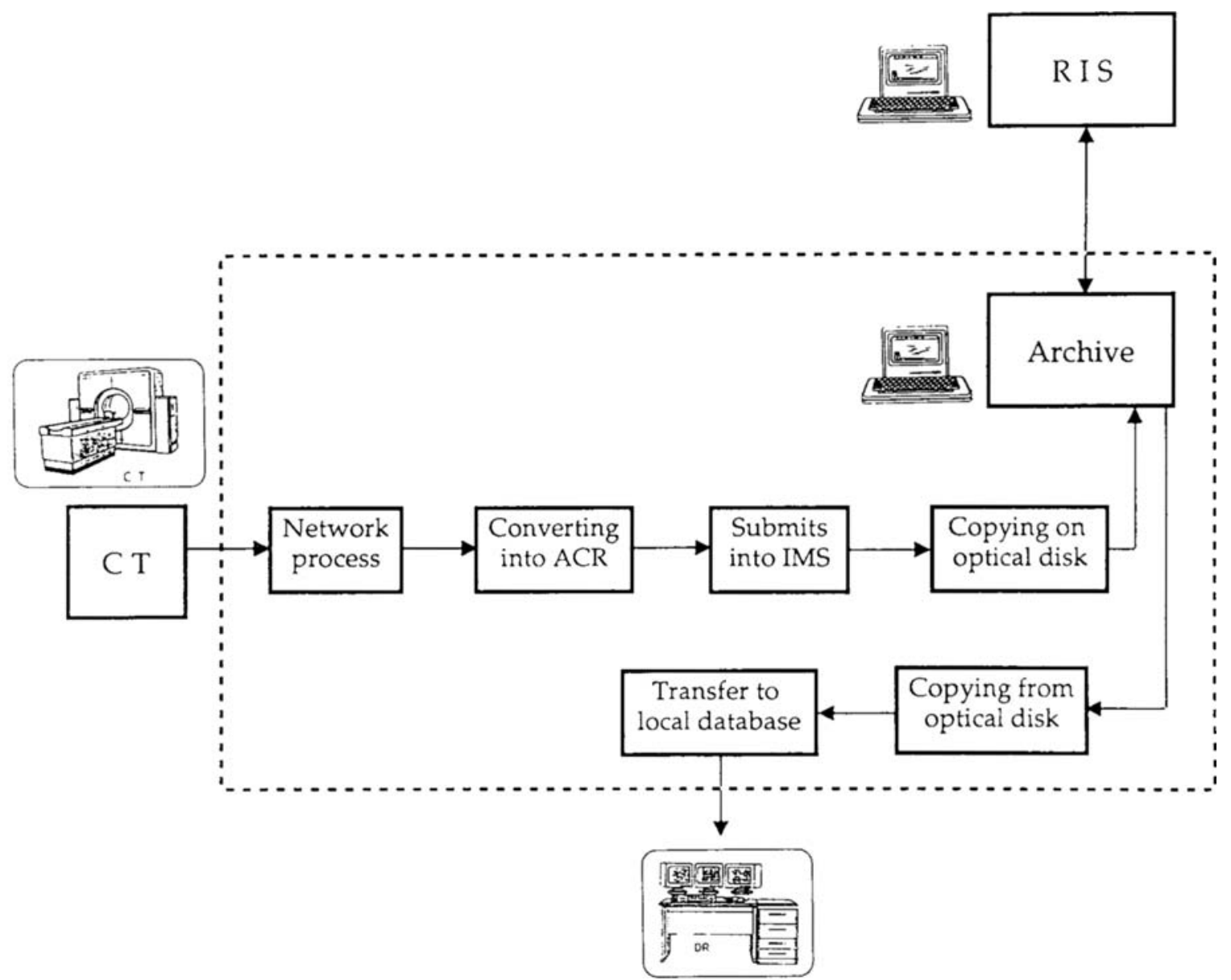

Fig 3. The PACS is organized as a group of software processes that communicate with each other. The sequence of the processes operates as a pipeline.

about the state of the system at any moment. The PACS-MONITOR enables the user to visualise the background activities of the PACS and the image flow within the PACS.

Structure. First, the PACS-Monitor gives the user overview information about which components (modalities, archive, consoles etc) are in use and which components are communicating with each other (archiving, retrieving etc).

In a second step, the user can obtain more detailed information: (1) the exact image flow on the different image directories; (2) the activities of the software processes in the background; (3) which examinations are submitted into the IMS; (4) which examinations are in the archive waiting queue-which examinations are in the retrieve waiting queue; and (5) from which examinations the disk identifications are still not entered in the RIS (either because they have not been reported or because it was impossible to add their identification to the RIS).

Via the PACS-MONITOR, the user can manipulate and manage the activities in the PACS. It is possible to perform the following exercises: (1) stop the archiving and retrieving; (2) restart the software processes (New Generation); (3) stop and restart the PACS (Initialization); (4) initialize the optical disk drives; and (5) configure the disk drives. To gain performance data, it is possible to display (for example at the end of the day shift) the storage allocations and process central processing unit times.

Function. With the PACS-MONITOR the user can check which tasks are already being executed by the PACS and which are still in a waiting queue. Furthermore, she/he can get information about the loading of the system, 


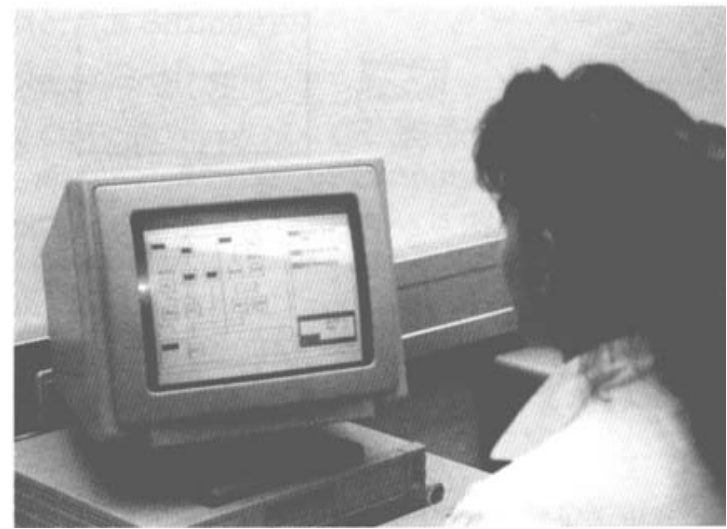

Fig 4. The PACS-MONITOR is used for the survey and management of the PACS.

which is of importance for the work organization. The PACS-MONITOR is menu-controlled and most information is displayed graphically. Some activities inside the PACS (for example: the activities of the software processes, the image flow etc) are checked automatically by the PACS-MONITOR at user defined time intervals.

\section{OPERAS}

OPERAS (OPERating ASsistent) is a rule based expert system for error detection and recovery in the PACS. It is an intelligent tool for the PACS user support. This expert system makes the routine operation as independent from the presence of an electronic data processing expert as possible.

Components. OPERAS consists of 3 main components: (1) A set of heuristic rules (object rules) for the localization and elimination of malfunctions, (2) Modules for the dialogue with the user and system interrogations, and (3) Metarules for the selection of tests. The set of heuristic rules contains rules for possible troubles in archive software, retrieve software, optical disk drives, etc. Depending on the initial information (eg, no CT scanner can transfer images to the archive, messages are still sent back from the archive), the metarules select different tests like archive network test, image directory test, and so on. At the moment, OPERAS contains 74 rules. An example of such a rule follows:

IF: it is possible to transfer images from other CT scanners
THEN: initialize the network process at your scanner first.

OPERAS session. OPERAS is started via the PACS-MONITOR. First the user indicates from a menu which function (archiving, retrieving, image transfer, etc) does not work. Then OPERAS collects information about the state of the PACS by opening a dialogue with the user and through system checks. From this information, one or more metarules is activated, a hypothesis about the possible reason of the malfunction is set up, and a group of object rules is selected to verify the hypothesis and to eliminate the malfunction. So a backward chaining is possible, and the troubles can be localized and eliminated in a few steps.

Either the program gives the user advice on how to eliminate the troubles or the troubles are eliminated automatically. At every moment of the session, the user can get help, explanations, and intermediate results from OPERAS. At the end of the session it is possible to get an analysis of all the activated metarules and object rules.

\section{PACS-View}

One of the critical points of a full PACS is the accessibility of images outside of the radiology department. PACS-View is an attempt to solve this problem by using the ubiquitous $\mathrm{PC}$ as a PACS terminal. Because we wanted to simply add image functionality to PCs that are already used for other purposes, we did not rely on any special hardware for enhanced image display. This approach differs from the one taken, for example, in the Department of Veteran's Affairs system where dedicated PC-based PACSstations with special graphic cards are used..$^{20}$

Structure. PACS-View is an image display console based on a $\mathrm{PC}$. The $\mathrm{PC}$ is equipped with an Ethernet card and video graphic array monitor. The software process that manages the database and communicates with the PACS process structure and the database itself is located in a VAX. The PC has access to the images on the VAX via PCSA (Network Operating Software). In a more sophisticated version, the PC is linked with an high-resolution $(1,024 \times 1,024)$ screen (IMLOGIX).

Function. The PACS-View serves as a PACS terminal that allows easy access to the images 


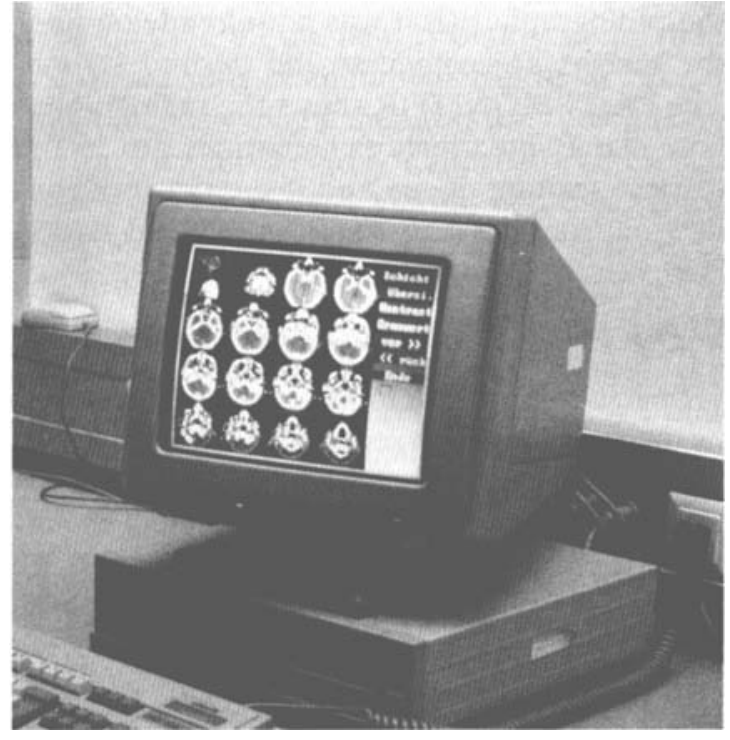

Fig 5. The PACS view is an image display console based on a PC.

for clinical departments. With the PACS-View software, every standard PC (with the Ethernet card and the VGA monitor) can be turned into a cheap PACS terminal. Image quality for digital images like CT or $\mathrm{MRl}$ is sufficient for the needs of clinicians.

Operation. The examination is selected from a list of available examinations. Then all of the images of the examination are displayed in an overview image. The images to be viewed are selected from the overview screen with the mouse. Then the selected images may be displayed on the PC or on the alternate IMLOGIX (Fig 5). A few image processing capabilities such as windowing and pixel lens are also available.

\section{PACS-RIS INTERFACE}

\section{Structure}

The PACS is interfaced with the RIS in such a way that the management of the optical disks is done via the RIS by using patient data to select and retrieve the images of an examination. The interface is realized with the aid of a few messages and requests that are sent over the network.

\section{Function}

After the images are copied on optical disk, the disk number of the images and the directory information about their location on the disk are sent to the RIS computer from the image archive and written into a temporary file. After the report text is inserted into the RIS by a secretary (the radiologist dictates the report text on a dictating machine), the disk number and the directory information are added to the report and deleted in the file. After that step, the images are managed by the RIS, and access to the images is only possible via the RIS.

The examination is selected by entering, eg, patient name and birth date at an RIS terminal. Then the RIS sends a message with the identification of the disk where the requested images are stored and the directory identification about the location on the disk to the archive and the number of the disk is displayed at the archive console. Figure 6 shows the data and image flow in the PACS-RIS in terms of the Marburg model $^{21}$ by using a diagram of the control view. Because our CT scanners are older models with an inflexible PACS interface, they are not able to accept patient identification data from the RIS. This data must be entered again at the console of the CT scanner before the archiving is initiated and errors may occur. If some

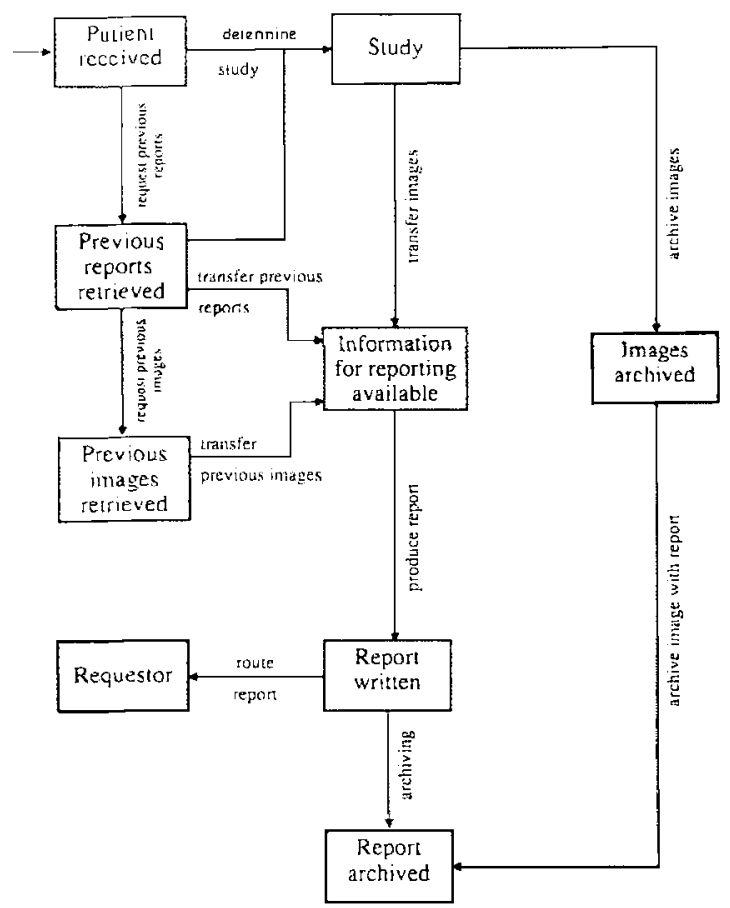

Fig 6. Control view diagram of the data and image flow in the PACS-RIS. 
patient data in the RIS and in the PACS do not correspond, the PACS-RIS interface compares other data, such as the examination number, and if they correspond, the disk number and the directory identification are entered into the RIS. If it is not possible to link the data automatically, it is possible to connect them manually.

\section{IMAGE AND DATA TRANSFER IN THE PACS}

Only a small amount of manual work is necessary for the different working procedures (archiving, retrieving, and image transfer) in the PACS. Most operations are done by the background software processes. During the execution of the working procedures, it is possible for the user to gain information about the sequence of operations via the PACS-MONITOR and to consult the OPERAS program if troubles occur.

\section{Archiving}

The radiographer initiates the archiving by entering patient data at the CT scanner and by selecting the station (archive), the function (send), and the number of images to be transferred. All the necessary operations (converting into ACR-NEMA, submitting into IMS, and copying on optical disk) are executed by the software processes in the background. After the images are copied on optical disk, the patient and examination data are printed on a line printer and a message is sent back to the CT scanner.

With the PACS-Monitor, the radiographer can check if the image flow is continuous or if there are any image accumulations in image directories. If the image transfer has been interrupted during the transmission, she/he can use the PACS-MONITOR to check how many images of the examination are already submitted into the IMS database and how many must be transferred once again. The radiographer can supervise data flow from the transfer over the network until the connection with the RIS.

\section{Retrieving}

The images are retrieved via the RIS by using patient identifiers. At an RIS terminal, the retrieval is initiated after selecting a report with patient name and birthdate. After insertion of the requested disk into the archive station by the radiographer (if it was not already on line) the images are copied to the desired station. The PACS-MONITOR shows the radiographer how many and which examinations are in the retrieve waiting queue and if the network connection from the archive to the selected station is successful.

\section{Image Transfer to the Viewing Stations}

Special CT or MRI examinations requested by the radiologist are sent to the PACS diagnostic console. During the image transfer AURA is inspected for previous examinations. The images from both the DSA and the archive (CT) can be sent to the image display console (DRC 101).

There also exists the possibility of transferring MRI examinations and $\mathrm{CT}$ examinations from the archive to the PACS-Viewing stations (PCs).

The PACS is not a stand-alone system that runs independently from the other equipment in the department. Failures that occur during the above listed working procedures can influence or block the routine work. One or more of the following may cause a failure: if a software process is not active, if the communication between two processes is interrupted, or if a hardware component fails after a power failure. OPERAS enables the radiographer to find and fix errors without calling on a computer expert.

\section{IMAGE PRODUCTION}

One optical disk can store up to 26,000 images with a resolution of $256 \times 256$ pixels, which corresponds to $1,000 \mathrm{CT}$ examinations. The storage of one year's image production (three CT scanners at the Department of Radiology, one CT scanner at the Department of Radiotherapy) requires 22 disks. (For more technical details about image production, system loading, and performance data see Gell. ${ }^{13}$ )

\section{EXPERIENCES}

\section{Application of PACS}

Our experience is that the PACS is very useful in the organizing of tasks for a department, especially for the procedures of archiving and retrieving of examinations.

The diagnostic console is not used routinely for primary diagnosis. The main reason seems 
to be that not all the images of an examination and of previous examinations can be displayed simultaneously on the monitors as is possible on the traditional light case or alternator.

We expect that image viewing consoles, which allow users from clinical departments a direct and fast access to the images, will be of clinical importance. Because the requester receives only electronic copies of the archived images, the original images are always available for other users (In the conventional film archive of our department, $25 \%$ to $30 \%$ of the films are not available when requested). There is also an increasing need of image exchange within the radiology department (CT, MRI, DSA, radiotherapy, nuclear medicine, etc). This is of special importance for integrated diagnosis.

\section{Routine Operations}

Because of the problem-free handling of the optical disks, there is a real saving of labor and time for the radiographer. The storage space required is much smaller than with magnetic tapes, and the handling of disks for storage and retrieval is more convenient and simpler than the handling of tapes. The time needed to archive and retrieve an examination is in accordance with the requirements of the department. The selection and retrieval of images via the RIS using patient identification allows a fast and reliable access to the images.

An important experience from routine work is that the confidence of the user in the PACS increases and the human error rate decreases because of the continuous availability of information in the system. This is of psychological importance too; as the PACS become easier to survey, the confidence of the user in the PACS increases.

Failures that occur during the routine work may spread and lead to impaired operations on parts or all of the system. The PACS runs 24 hours a day. To guarantee a smooth operation, it is important that failures be recovered immediately by the user himself. This is of special importance during the night and weekend shifts, when no EDP expert is present in the department. Because we have older (pre-PACS) CT scanners that are not able to collect patient data from the RIS or where the image header contains no information about the total number of images in the examination, we cannot yet connect the PACS and the RIS entirely to realize a completely autonomous software structure. For a discussion of the different requirements that an intelligent process structure with automatic supervision should conform see Wiltgen. ${ }^{22}$

\section{CONCLUSION AND OUTLOOK}

To the advantages of the RIS (sharing of data for patient care, fast access to the case history, etc) the PACS adds the advantages of a compact and easy to handle image archive, fast availability of images at clinical departments, and so on. We consider the PACS logically as a part of the RIS and, thus, the archived images are managed (in reduced form) as folders by the AURA database. The installation of a juke box is planned to be able to hold more examinations on line (the production of at least 1 year). To enable fast access to the images for the end users of the diagnostic information, PACS viewing stations will be installed at more clinical departments (neurosurgery, paediatric surgery, radiotherapy, etc). We are developing a tool that will allow an easy expansion of the PACS process structure in that sense that new processes (eg, administrative processes for local image data bases) can simply be added to or subtracted from the existing configuration without software changes in the existing process structure.

\section{REFERENCES}

1. Glass H: The impact of PACS on hospital information and practice, in Schneider H, Dwyer SJ, Jost RG (eds): Medical Imaging 3: PACS System Design and Evaluation, SPIE vol. 1093. Bellingham, WA, SPIE-The International Society for Optical Engineering, 1989, pp 354-361

2. Greinacher CFC, Bach EF, Herforth M, et al: Computer-assisted radiology requirements and solutions for digital diagnostic imaging. Med Inf 15, 1:21-29, 1990

3. Hindel R: PACS cost justification: An industrial perspective. Med Inf 15, 1:77-82, 1990
4. Passariello R, Agnifili A, Pavone P, et al: Functional integration of a radiological department: A pilot project, in Lemke HU, Rhodes ML, Jaffe CC, et al (eds): Computer Assisted Radiology, Proceedings of the International Symposium CAR 89. New York, NY: 1989, pp 510-514

5. ter Haar Romeny BM, van der Wielen JMM, Achterberg AJ, et al: PACS efficiency: a detailed quantitative study of the distribution process of films in a clinical environment in the Utrecht Univ Hospital, in Schneider H, Dwyer SJ, Jost RG (eds): Medical Imaging 3: PACS System Design 
and Evaluation, SPIE vol 1093. Bellingham, WA, SPIE-The International Society for Optical Engineering, 1989, pp 259-271

6. Van Poppel BM, Bakker AR, Wilmink JBM: A package for cost and critical analysis of picture archiving and communication indicating its true field (CAPACITY). Med Inf $15,1: 67-75,1990$

7. Glass $\mathrm{H}$ : The impact of PACS on hospital information and practice, in Schneider H, Dwyer SJ, Jost RG (eds): Medical Imaging 3: PACS System Design and Evaluation. SPIE vol 1093. Bellingham, WA, SPIE-The International Society for Optical Engineering, 1989, pp 354-361

8. Chan KK, Taira RK, Huang HK: Integration issues in PACS, in Huang HK, Ratib O, Bakker AR, et al (eds): Picture Archiving and Communication Systems. Nato ASI Series, vol F 74. New York, NY: Springer, 1991, pp 141-147

9. Bakker AR: HIS and RIS and PACS, in Huang HK, Ratib O, Bakker AR, et al (eds): Picture Archiving and Communication Systems. Nato ASI Series, vol F 74. New York, NY: Springer, 1991, pp 157-162

10. Giribona P: Principles for PACS evaluation, in Demongeot J, Sousa Pereira A (eds): ISCAMI 1: Integrated system for the Management and Manipulation of Medical Images. Paris, France, Springer, 1991, pp 16-23

11. Hruby W, Mosser H, Urban M, et al: The Vienna SMZO-PACS-Project: The totally digital hospital, in Lemke HU, Rhodes ML, Jaffe CC, et al (eds): Computer Assisted Radiology, Proceedings of the International Symposium CAR91. New York, NY, Springer, 1991, pp 436-441

12. Ottes FP, Bakker A, Kouwenberg JML: Are Hospitalwide PACS implementations possible now (soon)?, in Lemke HU, Rhodes ML, Jaffe CC, et al (eds): Computer Assisted Radiology, Proceedings of the International Symposium CAR91. New York, NY, Springer, 1991, pp 450-455

13. Gell G: AURA: A clinical data bank based on free text, in O'Moore RRO, Barber B, Reichertz PL, et al (eds): Lecture Notes in Medical Informatics, vol 16. New York, NY, Springer, 1982, pp 850-856
14. Gell G: Routine documentation of medical texts. Methods Inf Med 14:63-68, 1983

15. Madjaric M, Gell G: SYGMA-A general objectoriented medical application development system, in Adlassnig K, Grabner S, Bengtsson S, et al (eds): Medical Informatics Europe 1991. New York, NY, Springer, 1991, pp 127-131

16. Gell G, Schneider GH, Wiltgen M, et al: PACSPraktische Erfahrungen: Archivierung, Kommunikation, Befundung, in Schneider GH, Vogler E, Kocever K (eds): Digitale Bildgebende Verfahren-Interventionelle Verfahren--Integrierte digitale Radiologie, 6. Grazer Radiologisches Symposium, Graz 12.-14. Oktober 1989. Blackwell Ueberreuter Wissenschaft, Berlin, Germany, 1990, pp 536540

17. Wiltgen M, Gell G, Schneider GH: Application of a PACS in daily routine, in Huang HK, Ratib O, Bakker AR, Witte $G$ (eds): Picture Archiving and Communication Systems. Nato ASI Series, vol F 74, New York. NY, Springer, 1991, pp 431-434

18. Wiltgen M, Gell G, Schneider GH, et al: PACS: A pilot system in routine operation. Electromedia 58(4):124129,1990

19. ACR-NEMA Standards Publication/No 3001985 , Digital Imaging and Communications.

20. Dayhoff E, Maloney DL, Shepard BM: Integration of images with the Veterans Affairs Hospital information system in a distributed environment, in Kingsland LC (eds): Symposium on Computer Applications in Medical Care, Washington, DC, November 1989. IEEE Computer Society Press, Washington, Los Alamitos, Brussels, Tokyo, 1989, pp 971-974

21. Rienhoff O, Greinacher CFC: A general PACS-RIS interface, in Reichertz PL, Lindberg DAB (eds): Lecture Notes in Medical Informatics 37. New York, NY, Springer, 1988, pp 26-38

22. Wiltgen M, Gell G, Schneider GH: Some software requirements for a PACS: Lessons from experiences in clinical routine. Int J Biomed Comput 28:61-70. 1991 